\title{
Physical Origins of Universal Mobility in MOSFET Inversion Layers: Conservation Laws
}

This paper was downloaded from TechRxiv (https://www.techrxiv.org).

\section{LICENSE}

CC BY 4.0

SUBMISSION DATE / POSTED DATE

$24-12-2021$ / 29-12-2021

\section{CITATION}

Shoucair, F. (2021): Physical Origins of Universal Mobility in MOSFET Inversion Layers: Conservation Laws. TechRxiv. Preprint. https://doi.org/10.36227/techrxiv.17570168.v1

DOI

10.36227/techrxiv.17570168.v1 


\title{
Physical Origins of Universal Mobility in MOSFET Inversion Layers: Conservation Laws ${ }^{1}$
}

\author{
F. S. Shoucair
}

\begin{abstract}
The salient properties of charge flow (or current) along the MOSFET's inversion layer are shown to be consilient with a river's flow in a gravitational potential field, insofar as both are fundamentally governed by energy conservation principles, and their laminar and turbulent conditions determined by friction losses at shallow depths. We establish analytically that the low-field, "universal" effective, mobility $\mu_{\text {eff }}$, long reported to vary as $\sim\left(E^{*} T\right)^{-1 / 3}$ for transversal fields below $0.5 \mathrm{MV} / \mathrm{cm}$, is manifestation and consequence of both energy and momentum conservation under laminar flow conditions and quantum mechanical effects, in which case the inversion layer's mean thickness $\langle z\rangle$ also varies as $\sim\left(E^{*} T\right)^{-1 / 3}$ up to a maximum value $E^{*_{T}} \approx 0.35 \mathrm{MV} / \mathrm{cm}$ at $300 \mathrm{~K}$, determined only by interface "terrain" amplitude and fundamental constants.
\end{abstract}

Physics is mathematical, not because we know so much about the physical world, but because we know so little: it is only its mathematical properties that we can discover. [1]

\section{INTRODUCTION}

The effective mobility, $\mu_{\text {eff }}$, is a parameter of prime importance among the characteristics underlying the behavior of the MOSFET's (Metal Oxide Semiconductor Field Effect Transistor) inversion layer, or current carrying channel. In modern, downscaled, devices, whose gate oxides are near to, and below, $t_{o x} \approx 20 \AA, \mu_{\text {eff }}$ is observed to fall more steeply with increasing $E^{*} T$ than expected from its "universal" behavior, whereby $\mu_{\text {eff }}$ varies as $\sim\left(E^{*} T\right)^{-1 / 3}$ [2], [3], which devices with thicker oxides and inversion layers have displayed over many prior technological generations. This mobility degradation is widely attributed to the growing, and increasingly dominant, influence of irregularities and/or "scattering" effects occurring at the $\mathrm{Si} / \mathrm{Gate}$-insulator interface, as $E^{*_{T}}$ exceeds $0.5 \mathrm{MV} / \mathrm{cm}$ approximately in tandem with higher bulk doping concentrations, higher gate insulating dielectric constants, and thinner gate oxides, among other evolving physical parameters, as entailed by downscaling. Consequently, accurate models of inversion layers of extents comparable to a single silicon inter-atomic distance $(\approx \pm 5 \AA)$ are required towards further developments, not only because their deleterious effects increasingly encroach upon critical MOSFET performance, but because the latter' complex interactions are likely further to complicate the task of disentangling their respective influences as well.

In section II, we establish the similar general relationships pertaining to a river's flow under gravitation, and to the MOSFET's current flow under low longitudinal field conditions, which relate the depths $z$ of the respective "fluids" to the relevant parameters of these systems. These considerations readily lead to the origins of the behavior of "universal mobility", $\mu_{\text {eff }} \sim\left(E^{*}\right)^{-1 / 3}$, as a consequence of laminar flow, and energy and momentum conservation under quantum-mechanical uncertainty relationships, as elaborated in II. $C$,

\footnotetext{
${ }^{1}$ This is a self-contained, abbreviated, version of sections I and II (only) of the manuscript entitled "Conservation Laws at Physical Origins of Universal Mobility in MOSFET Inversion Layers in Consilience with River Flow in a Gravitational Field" (https://doi.org/10.36227/techrxiv.17088419.v1), recast in standalone form. Sections III through VI are similarly archived under the title "Modeling the MOSFET's Inversion Layer and its Universal Mobility: A New Experimental Method". ${ }^{2}$ The Greek letter $\mu$ and the Roman letter M of our modern alphabet originate from the Egyptian hieroglyphic symbol $\wedge \wedge \wedge \wedge$ for water.
} 
along with a maximum value for $E^{*} T$ beyond which laminar flow ceases.

The analyses to follow aim to establish such relationships between the aforementioned phenomena as derivable from first principles. We thus bring conservation of energy and momentum laws to bear upon these from the outset, which are seldom, if at all, invoked explicitly in this context. This approach, thus underpinned and framed by a scaffolding of fundamental principles, provides not only a variant pedagogical perspective on the complex effects attending the characteristics of the MOSFET's inversion layer, but such foundational safeguards as well, as are vital to assessing the validity of prospective physical constructs and interpretations thereof, especially in light of a considerable body of (often disparate) experimental observations extant in the literature - our reference section listing a mere representative sample thereof.

\section{PARTIAL ANALOGY BETWEEN THE FLOW OF INVERSION LAYER ELECTRONS AND THAT OF A RIVER IN A GRAVITY FIELD, BOTH CONSIDERED FROM THE PERSPECTIVE OF ENERGY $(\mathcal{E})$ CONSERVATION}

The variable $z$ represents depth of river and depth (thickness) of inversion layer, as indicated in Fig. 1.
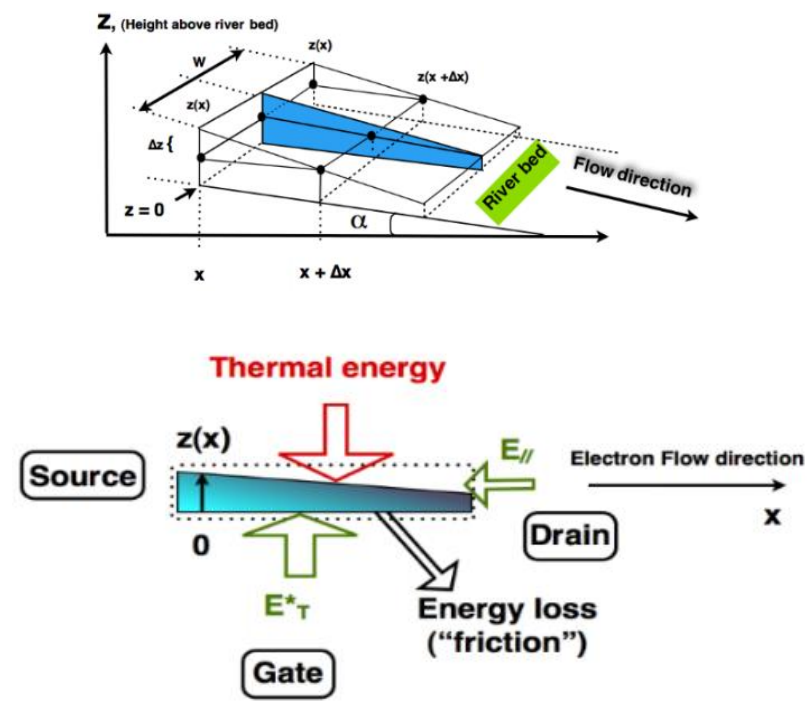

Fig. 1. Top: View of a wide $(W)$ river of depth $z(x)<<$ $W$ above its bed, flowing in the positive $x$ direction at an angle $\alpha$, the slope of the bed to the horizontal.

Bottom: Cross sectional view of an inversion layer's volume: electrons flow in the positive $x$ direction, from source towards drain. The "height" of an electron above the "floor" level (the gate oxide plane) is the thickness of the inversion layer $z(x)<<W$; the direction of $W$ is not shown. The ratio $\left(E_{/ / /} E^{*} T\right)$ corresponds to the slope of the "riverbed" above, with $E_{/ /}$the longitudinal electric field. The wide arrows represent various energies entering or leaving the volume, only some of which are conserved.

\section{A. River [4]}

The gravitational potential energy difference (loss) between depths $z(x)$ and $z(x+\Delta x)$ above the bed is $m g \Delta z$, where $(\Delta z / \Delta x)$ $=\tan \alpha$, and $(1 / 2) m\left(v_{\text {drift }}\right)^{2}$ is the kinetic energy gain between $x$ and $(x+\Delta x)$. Energy loss to friction, or viscosity, along the riverbed is proportional to kinetic energy, and inversely to depth $z(x)$. The coefficient of hydraulic friction $K_{F} \geq 0$, is a proportionality constant between the latter quantities. While potential and kinetic energies are conserved, energy lost to friction is not, and we have:

$$
\frac{\Delta}{\Delta x}\left[\mathcal{E}_{\text {potential }}+\mathcal{E}_{\text {kinetic }}\right]+\mathcal{E}_{\text {friction }}=0
$$

hence,

$$
\begin{gathered}
\frac{\Delta}{\Delta x}\left\{m g[z(x)-\Delta x \tan \alpha]+\frac{1}{2} m v_{\text {drift }}^{2}\right\}+ \\
{\left[\frac{K_{F}}{z(x)}\right] \frac{1}{2} m v_{\text {drift }}^{2}=0}
\end{gathered}
$$

dividing through by $m g$ and taking the limit of the differences readily yields:

$\frac{d}{d x}\left\{z(x)+\frac{1}{2 g} v_{d r i f t}^{2}\right\}-\tan \alpha+\left[\frac{K_{F}}{z(x)}\right] \frac{1}{2 g} v_{d r i f t}^{2}=0$

If the flow of the river is steady at a mean velocity $v_{\text {drift, }}$, such that $Q$ is the volume, assumed constant, flowing across a given cross-section per unit of time, the following continuity equation holds:

$$
z(x) \cdot v_{\text {drift }}=Q
$$


Substituting $v_{\text {drift }}$ from (4) into (3) yields the slope $(d z / d x)$ as a function of $z(x)$ :

$$
\frac{d z}{d x}=\tan \alpha\left[\frac{1-\left(\frac{K_{F} Q^{2}}{2 g \tan \alpha}\right) \frac{1}{z(x)^{3}}}{1-\left(\frac{Q^{2}}{g}\right) \frac{1}{z(x)^{3}}}\right]
$$

The relative magnitudes of $K_{F}$ and $\alpha$ determine the magnitude and sign of $(d z / d x)$, and the integral curves of (5) yield the profile $z(x)$ of the free surface.

Isoclines are straight lines parallel to the bed, which correspond to constant values of $(d z / d x)$, a stream of uniform depth, laminar flow, and a river flowing into a reservoir. When $z(x)$ is large enough so that the ratio inside the square brackets is near to unity, the flow is parallel to the slope of the riverbed, since $(d z / d x) \approx \tan \alpha$.

If the ratio inside the square brackets changes sign, at shallow depths where $z(x)$ is small for example, the flow may incur discontinuities in height and velocity, or hydraulic "jumps", thereby becoming turbulent or chaotic, such that the river becomes a torrent.

The case $(d z / d x)=0$, corresponding to a level surface, or to a fluid in equilibrium at the bottom of a bucket, was previously invoked by C. Séquin [5], to promote visualization of the behavior of certain chargecontrolled devices, including charge-coupled devices (CCD's) and MOSFETs, whereas non-laminar flow conditions were indicated as pertinent to MOSFETs operating in saturation, where they are typically biased in analog circuits. The latter conditions entail more complicated dynamics [6], beyond the purview of the present analyses.

\section{B. Inversion Layer}

The potential energy difference for an electron traveling an incremental distance $\Delta x$ along the channel in the positive $x$ direction, i.e., from source towards drain and "falling" a distance $\Delta z$ in the positive $z$ direction inside the inversion layer (i.e., from the bulk towards the gate's "attractive" potential) is:

$$
\begin{gathered}
q[V(x)-V(x+\Delta x)]=q \Delta V= \\
-q\left(E_{T}^{*} d z+E_{I I} d x\right)
\end{gathered}
$$

For an inversion layer charge density varying nearly linearly with $z$, i.e., $\rho(z) \sim z$, which we establish from experimental observations of the ratio of mean to surface potentials $\left(\psi * / \psi_{s}\right)$ in [7], and consistently with the time-independent solution of Schrodinger's equation given the boundary conditions of the system [9]:

$$
\rho(z) \approx \rho_{o}\left(\frac{z}{z_{\max }}\right)^{m}, \text { with } \mathrm{m} \approx 1
$$

we have, equivalently to the continuity (4) for the river:

$$
\begin{aligned}
I_{D}=\frac{d Q_{i}}{d t} & =\frac{d}{d t}\left[\frac{1}{2} q\left(\frac{\rho_{o}}{z_{\max }}\right) W z(x) d x\right] \\
& =\frac{1}{2} q\left(\frac{\rho_{o}}{z_{\max }}\right) W z(x) v_{\text {drift }}
\end{aligned}
$$

wherein $Q_{i}$ is the total mobile charge in a cross- sectional slice $d x$ of the inversion layer, and the constant drain current $I_{D}$, flowing at a mean velocity $v_{\text {drift }}$, is the counterpart of the river's constant volume $Q$ flowing per unit of time and width. In this case:

$$
v_{\text {drift }} \approx\left(\frac{2 I_{D} Z_{\max }}{q \rho_{o} W z(x)}\right)=\mu_{e f f} E_{I I}
$$

is the "classical" counterpart of (4), whose value is approximately $250 \mathrm{~m} / \mathrm{s}$ (when $\mu_{\text {eff }} \approx$ $\left.500 \mathrm{~cm}^{2} / \mathrm{V} \cdot \mathrm{s}, E_{/ /} \approx 50 \mathrm{~V} / \mathrm{cm}\right)$, near to the speed of sound in dry air at standard temperature and pressure, for $\left(I_{D} / W\right) \approx 0.1 \mu \mathrm{A} / \mu \mathrm{m}, z(x) \approx 20 \AA$, and $\left(\rho_{o} / z_{\text {max }}\right) \approx 10^{24} \mathrm{~m}^{-3}$, the latter volume density corresponding to a surface density of $\approx 10^{12} \mathrm{~cm}^{-2}$ for a commensurate charge-sheet model (see [7], Fig.2). 
Proceeding as in the river case, the following statement of conservation of energy:

$$
\begin{gathered}
-E_{T}^{*} \frac{d z}{d x}-E_{I I}+\frac{m}{2 q}\left(\frac{2 I_{D} z_{\max }}{q \rho_{o} W}\right)^{2} \frac{d}{d x}\left[\frac{1}{z(x)}\right]^{2}- \\
\frac{K_{F}}{z(x)}\left(\frac{m}{2 q}\right)\left(\frac{2 I_{D} z_{\max }}{q \rho_{o} W}\right)^{2}\left[\frac{1}{z(x)}\right]^{2}=0
\end{gathered}
$$

may readily be put in the form:

$$
\frac{d z}{d x} \approx-\left(\frac{E_{I I}}{E_{T}^{*}}\right)\left[\frac{1+K_{F}\left(\frac{m}{2 q E_{I I}}\right)\left(\frac{2 I_{D} Z_{\max }}{q \rho_{o} W}\right)^{2} \frac{1}{z(x)^{3}}}{1+\left(\frac{m}{2 q E_{T}^{*}}\right)\left(\frac{2 I_{D} Z_{\max }}{q \rho_{o} W}\right)^{2} \frac{1}{z(x)^{3}}}\right]
$$

and is the counterpart of (5) in the foregoing river case. Equation (11) thus lends itself to the following interpretations:

a) Large $z$ (thick inversion layer $\sim$ deep river): $(d z / d x) \approx-\left(E_{/ / /} / E^{*} T\right)$, which is the familiar "gradual channel" approximation when the ratio $\left(E_{/ / /} / E^{*} T\right),<<1$, as typically the case for long channel devices, and is in the range of $(1 / 2000)$ to $(1 / 6000)$ for our data [7].

b) Small $z \rightarrow 0$ (thin inversion layer $\sim$ shallow river): $(d z / d x)$ tends to the limit $\left(-K_{F}\right)$, which indicates that electron flow is predominantly determined by surface scattering conditions, with $K_{F}$ corresponding to "friction" along the insulating interface (the "riverbed"). Hence the magnitude of $(d z / d x)$ may vary locally, along $x$ if $K_{F}$ does so, such that a "gradual" channel no longer obtains along the entire length of the conducting channel. If, furthermore, $\left|K_{F}\right|$ should be sufficiently large, or (worse), a function increasing as a power of $(1 / z)$, then $(d z / d x) \approx-K_{F}(x, z)$ would behave in like manner, thereby yet again invalidating a "gradual" channel assumption and, importantly, result in mobility decreasing at a rate other than universal curves' power of $\left(E^{*} T\right)^{-1 / 3}$. Such phenomena are observable in [3,I, Figs.1-5] for electrons and holes under both low temperature and high $E^{*} T$ conditions.

c) The "pinch-off" condition corresponds to the latter situation whereby, in view of current continuity, the velocity of carriers along the channel (between source and pinch-off point) may vary as dictated by $K_{F}(x, z)$, while that between the pinch-off point and the drain increases owing to the local, accelerating, longitudinal field $E_{/ / /}$. Indeed, velocity "saturation" is observed under these circumstances, whose value is near to $10^{7} \mathrm{~cm} / \mathrm{s}$ at $300 \mathrm{~K}$ for electrons and holes on $<100>$ silicon surfaces [8].

d) Laminar flow, with $z(x)$ not restricted to large values, occurs along isoclines, or curves along which $(d z / d x)$ is constant: (5) and (11) may be solved for constant values of $(d z / d x)$. In the case of special interest whereby $\left|E_{/}\right|<<\mid E^{*} T$, and the effects of $K_{F}$ negligibly small, the result is proportionality between $z$ and $\left(E^{*} T\right)^{-1 / 3}$, which is therefore consequence of our starting premise $\rho(z) \sim z$, per (7). While the expression for the quantummechanical, average, extension $\left\langle z_{Q M}>\right.$ derived by Stern and Howard [9] resulted from an ostensibly different function, $\rho(z) \sim z^{2} e^{-\beta z}$, yet they obtained very nearly the same functional relationship $\left\langle z_{Q M}>\sim\left(E^{*} T\right)^{-1 / 3}\right.$, by solving the timeindependent Schrodinger's equation subjected to the boundary conditions of the system.

\section{Similarities and Distinctions between River and Inversion Layer Cases}

The foregoing analogy between the river and inversion layer cases is apposite though limited, because the density of the "incompressible fluid" in the former is considerably higher than that of electrons, whose volume density is more akin to that of a diffuse "gas". Since, moreover, the motion of electrons in the inversion layer is governed by Schrödinger's equation as constrained by the boundary conditions of the system [9], this 
motion may be construed as the diffusion of a probability amplitude from one point to the next along the $x$ axis. Whereas ordinary diffusion of a gas along a thin tube gives rise to real exponential functions, Schrödinger's equation yields complex waves for an "oscillating gas" [10],[12]. The respective $K_{F}$ parameters, constant or otherwise, can thus be expected to be different, qualitatively and quantitatively, for the two cases.

\section{1) Dependence of inversion layer thickness on $E^{*} T$ and $T$}

To the extent that the inversion layer may be regarded, conceptually, as the combination of a classical $\left(z_{c l}\right)$ and a quantummechanical $(Z Q M)$ component [11], the following relationships obtain:

$$
\frac{3}{2} k T=q E_{T}^{*} z_{c l}=\frac{1}{2} m v_{t h}^{2}
$$

where $(3 / 2) k T$ is the average translational kinetic energy of a molecule of an ideal gas at a given temperature $T$, with $k$ Boltzmann's constant, hence

and

$$
Z_{c l}=\frac{3}{2} \frac{k T}{q} \frac{1}{E_{T}^{*}}
$$

$$
v_{t h}=\sqrt{\frac{2 q E_{T}^{*} z_{c l}}{m}}=\sqrt{\frac{3 k T}{m}}
$$

is the (rms) value of the thermal velocity $v_{\text {th }} \approx 1.15 \times 10^{5} \mathrm{~m} / \mathrm{s}>>v_{\text {drift }} \leq 250 \mathrm{~m} / \mathrm{s}$ at $300 \mathrm{~K}$, [7], whereas the quantum-mechanical component $z Q M$ resulting from the uncertainty relationship $(\Delta p \Delta z) \geq \hbar$, where $\hbar=h / 2 \pi$, with $h$ Planck's constant, associated with the wave nature of electrons [10],[12], yields:

$$
\frac{p^{2}}{2 m}=\frac{1}{2 m}\left(\frac{\hbar}{z_{Q M}}\right)^{2}=q E_{T}^{*} z_{Q M}=\frac{1}{2} m v_{Q M}^{2}
$$

which may be construed as an energy term when put in a form similar to (12a), such that

$$
z_{Q M}=\left[\frac{\hbar^{2}}{2 m q E_{T}^{*}}\right]^{\frac{1}{3}}
$$

is the counterpart of (12b), and a velocity

$$
v_{Q M}=\sqrt{\frac{2 q E_{T}^{*} z_{Q M}}{m}}=\frac{\hbar}{m z_{Q M}}
$$

the counterpart of (12c). The conservation of energy $(\mathcal{E})$ constraint at fixed $x$ and $T$, for $z_{c l} \approx z_{Q M} \approx z:$

$$
\frac{d}{d z}\left[q E_{T}^{*} z+\frac{1}{2 m}\left(\frac{\hbar}{z}\right)^{2}\right]=0
$$

thus yields the approximate corresponding value of $z$ :

$$
\left.z\right|_{(\Delta \mathcal{E}=0)} \approx\left[\frac{\hbar^{2}}{m q E_{T}^{*}}\right]^{\frac{1}{3}}
$$

which varies as $\left(E^{*}\right)^{-1 / 3}$ and, unlike the river case, is subject to the minimum value imposed by the uncertainty relationship implicit in (13a), as accounted for in the next subsection, and without which (14a) would allow arbitrarily small $z$ with increasing $E^{*} T$.

2) Maximum value of $E^{*} T$ for which laminar flow may be expected

The same uncertainty relationship $\Delta p \Delta z \geq \hbar$ associated with momentum

$$
p=m v_{t h}=\sqrt{3 m k T}
$$

in the $z$ direction in turn yielding:

$$
\Delta z \geq \frac{\hbar}{\sqrt{3 m k T}} \approx 10 \AA
$$

at $300 \mathrm{~K}$, the inversion layer's thickness may be expected to "clear" the Si/oxide interface terrain (see Fig.2a) when:

$$
\begin{aligned}
&\left.z\right|_{(\Delta \mathcal{E}=0)}-\Delta z \approx\left[\frac{\hbar^{2}}{m q E_{T}^{*}}\right]^{\frac{1}{3}}-\frac{\hbar}{\sqrt{3 m k T}} \geq \\
& A_{o}=\text { amplitude of interface terrain }
\end{aligned}
$$

hence when

$$
\left|E_{T}^{*}\right| \leq E_{T M A X}^{*}\left(A_{o}, T\right) \approx \frac{\hbar^{2}}{m q\left[A_{o}+\frac{\hbar}{\sqrt{3 m k T}}\right]^{3}}
$$

which may serve as an upper limit estimate for laminar flow under ideal conditions of negligibly small $K_{F}$, and is a function only of 
terrain roughness amplitude $A_{o}$ and fundamental constants. Figure 2(a) displays the variations of $z_{c l}$ per (12b), $z_{Q M}$ per (13b), $\left.z\right|_{(\Delta \mathcal{E}=0)}$ per (14a), and $\Delta z$ per $(14 \mathrm{~b})$; the vertical arrows located at $\left(E^{*} T\right)_{M A X}(300 \mathrm{~K}) \approx$ $0.34 \mathrm{MV} / \mathrm{cm}$ occur where $\left.z\right|_{(\Delta \mathcal{E}=0)}-\Delta z \approx$ $15 \AA-10 \AA=5 \AA$ just "clears" a terrain of amplitude $A_{o} \approx 5 \AA$, commensurate with silicon's lattice dimensions and with the $\pm 5 \AA$ interface amplitude observed for thermally oxidized $<100>$ surfaces.

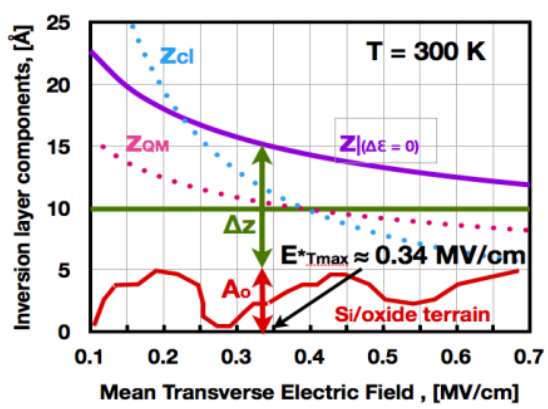

Fig. 2(a) Variations of $z_{c l}$ per (12b), $z_{Q M}$ per (13b), $\left.z\right|_{(\Delta \mathcal{E}=0)} \operatorname{per}(14 \mathrm{a})$, and $\Delta z$ per $(14 \mathrm{~b})$, at $300 \mathrm{~K}$.

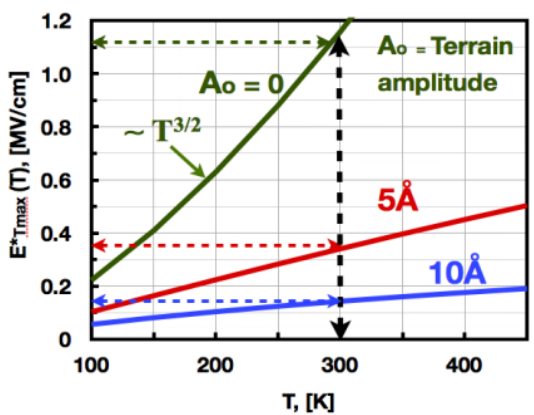

Fig. 2(b) Upper limit $\left(E^{*} T\right)_{\operatorname{MAX}}(300 \mathrm{~K}) \approx 0.34 \mathrm{MV} / \mathrm{cm}$ predicted by (15), above which laminar flow ceases.

The maximum electric field's value expected from (15), for $A_{o}=0$ at $300 \mathrm{~K}$ is near to $1.16 \mathrm{MV} / \mathrm{cm}$, as indicated by the dashed arrows in Fig.2(b).

Figure 2(c) shows the unconstrained value of the inversion layer per (14a) (dashed curve), under the ideal condition $A_{o}=0$, along with the constraints imposed by the uncertainty relationship (14b) at $T=100 \mathrm{~K}$, $300 \mathrm{~K}$, and $400 \mathrm{~K}$ (solid horizontal lines).
As far as may be discerned from their published figures [3,I, Figs.4,5], (15) appears to be in quantitative agreement with Takagi et al's data with respect to the temperature dependence of this maximum value for $E^{*} T$, namely $\sim T^{3 / 2}$ in the limit $A_{o}=0$, and qualitatively as to its relative insensitivity to the different masses of electrons and holes, and to surface orientations [3,II].

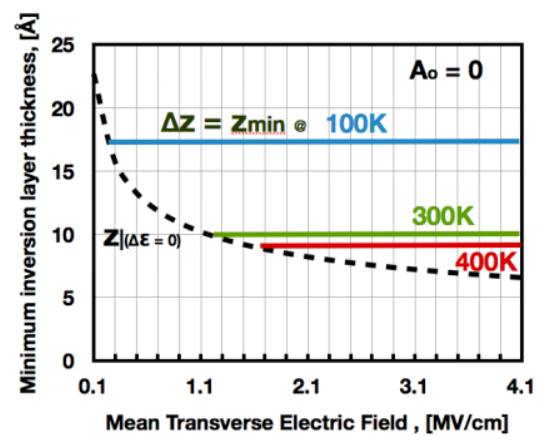

Fig. 2(c) Minimum extent of inversion layer.

Equation (15)'s close agreement with our observations is otherwise readily apprehended in Figs. 1-3, and 5, in [7], wherein our experimental results are further elaborated.

3) Establishing that the variations of $\mu_{\text {eff }}$ as $\sim\left(E^{*}\right)^{-1 / 3}$ result from conservation laws, laminar flow conditions, and quantummechanical effects

To the total energy $\left[q E^{*} T\left(z_{c l}+z_{Q M}\right)\right]$, which is the sum of (12a) and (13a), we may ascribe the equivalent velocity $v_{e q} \sim v_{t h} \sim$ $v_{Q M} \gg v_{\text {drift }}$ :

$$
v_{e q}=\sqrt{\frac{2 q E_{T}^{*}\left(z_{c l}+z_{Q M}\right)}{m}}
$$

Momentum (strictly only the $x$ component thereof), and energy conservation within an incremental inversion layer volume then yield:

and

$$
m\left(v_{\text {drift }}+v_{\text {eq }}\right)=\text { constant }
$$

$$
\begin{gathered}
m\left(v_{\text {drift }}^{2}+v_{e q}^{2}\right)= \\
m\left[\left(v_{\text {drift }}+v_{\text {eq }}\right)^{2}-2 v_{\text {drift }} \cdot v_{\text {eq }}\right]=\text { constant }
\end{gathered}
$$


wherein the first term inside the square brackets is constant, per (17). Since $v_{d r i f t}=$ $\left(\mu_{\text {eff }} E_{/ /}\right)$, it follows that the product:

$$
\left(v_{\text {drift }} \cdot v_{e q}\right)=\mu_{\text {eff }} E_{I I} \sqrt{\frac{2 q E_{T}^{*}\left(z_{c l}+z_{Q M}\right)}{m}}
$$

must be constant. Since $v_{e q}$ corresponds nearly to the total energy in the inversion layer's volume, for which $z \sim\left(E^{*} T\right)^{-1 / 3}$ established by (14a) is the condition for laminar flow per II.B.d) above - consistently with the solution of Schrödinger's equation as constrained by the boundary conditions of the system [9] the product $\left[E^{*} T\left(z_{c l}+z_{Q M}\right)\right]$, under the radical in (19) varies as $\sim E_{T}^{*}\left(E_{T}^{*}\right)^{-1 / 3} \sim\left(E_{T}^{*}\right)^{2 / 3}$ which, in turn, requires:

$$
\mu_{\text {eff }}\left(E_{T}^{*}\right)^{1 / 3}=\text { constant }
$$

so that $\mu_{\text {eff }}$ varies as $\sim\left(E^{*} T\right)^{-1 / 3}$, as is indeed the reported behavior of "universal mobility" curves for $\left(E^{*} T\right) \leq 0.5 \mathrm{MV} / \mathrm{cm}$, for both electrons and holes [2],[3]. The concordance between the foregoing results is essentially owed to the equivalence between our starting premise (7), and the solution of Schrödinger's equation [9] which embodies conservation laws.

\section{SUMMARY}

We have elucidated physical origins of the dominant phenomena governing current conduction in the MOSFET's inversion layer, below saturation, by comparing the latter to a broad river's flow in a gravitational potential field, whereby energy conservation considerations are fundamental. We thus established:

(1) Laminar current flow and maximum drift velocity result from energy conservation, concurrently with the gradual channel approximation entailing $\langle z\rangle$ varying as $\sim\left(E^{*} T\right)^{-1 / 3}$.

(2) The behavior of "universal mobility" curves, whereby $\mu_{\text {eff }}$ also varies as $\sim\left(E^{*} T\right)^{-1 / 3}$, is consequence and manifestation of laminar flow conditions.
We established this result, analytically, by invoking energy and momentum conservation, as well as the prevalence of quantum-mechanical effects.

(3) An analytical expression for an upper limit to the transverse electric field for which laminar flow may be expected, $\left(E^{*} T\right)_{M A X}(300 \mathrm{~K}) \approx 0.35 \mathrm{MV} / \mathrm{cm}$, which is a function of $\mathrm{Si}$ /oxide interface "terrain roughness" amplitude and fundamental constants only, in quasi-quantitative agreement with observations extant in the literature with respect to temperature and surface orientation for both electrons and holes.

\section{ACKNOWLEDGMENT}

The author is indebted to Mrs. V. Vigil, Berkeley Public Library, Berkeley, CA, and to R. Rice, for valuable technical discussions and assistance.

\section{REFERENCES}

[1] B. Russell, An outline of Philosophy, Meridian Books, New York, 1960, p.163.

[2] S. C. Sun and J.D. Plummer, "Electron mobility in inversion and accumulation layers on thermally oxidized silicon surfaces", IEEE Trans. Electron Devices, vol. ED-27, no.8, pp. 1497-1508, August 1980.

[3] S. Takagi, A. Toriumi, M. Iwase, and H. Tango, "On the universality of inversion layer mobility in $\mathrm{Si}$ MOSFET's: Part - I Effects of substrate impurity concentration, Part - II, Effects of surface orientation", IEEE Trans. Electron Devices, vol. ED-41, no.12, pp. 2357-2368, December 1994.

[4] T. Von Karman and M. A. Biot, Mathematical Methods in Engineering, McGraw-Hill, New York and London, 1940, pp.111-161.

[5] C. Séquin, in C. Mead and L. Conway, Introduction to VLSI systems, Addison Wesley, 1980, p.29.

[6] M. Lundstrom and Z. Ren, "Essential physics of carrier transport in nanoscale MOSFETs", IEEE Trans. Electron Devices, vol. ED-49, no.1, pp.133-141, January 2002.

[7] F. S. Shoucair, "Modeling the MOSFET's Inversion Layer and its Universal Mobility: A New Experimental Method", unpublished. [Online]. Available:

https://doi.org/10.36227/techrxiv.17088419.v1

[8] S. M. Sze, Physics of Semiconductor Devices, John Wiley \& Sons, 1981, p.46.

[9] F. Stern and W.E. Howard, "Properties of semiconductor surface inversion layers in the electric quantum limit", Physical Review, vol. 163, no.3, pp. 816-835, November 1967. 
[10] R. P. Feynman, R. B. Leyton, M. Sands, The Feynman Lectures on Physics, vol. III, Quantum Mechanics, Addison-Wesley publishing company, 1966, ch.16, pp. 8-16.

[11] S. A. Schwarz and S. E. Russek, "Semi-empirical equations for electron velocity in Silicon: Part II MOS inversion layers “, IEEE Trans. Electron Devices, vol. ED-30, no.12, pp. 1634-1639, December 1983.

[12] H. D. Young, University Physics, Addison-Wesley, 1992, pp. 1140-1182. 